\title{
AVALIAÇÃO DA MATÉRIA ORGÂNICA E pH DO SOLO EM SISTEMAS AGROFLORESTAIS LOCALIZADOS NA REGIÃO DE ALTAMIRA-PA
}

\author{
Sandra Andréa Santos da Silva ${ }^{1}$, Ana Claudia Moraes e Silva ${ }^{2}$, Daniel Baía \\ Gonçalves ${ }^{3}$, Fábio Miranda Leão ${ }^{4}$. \\ ${ }^{1}$ Prof ${ }^{a}$ Doutora, Adjunto II, da Universidade Federal do PARÁ - UFPA, Campus \\ de Tucuruí. E-mail: sandrasilva@ufpa.br \\ 2 Eng. Agrônoma, Universidade Federal do PARÁ - UFPA, Campus de Altamira. \\ ${ }^{3}$ Graduando em Engenharia Sanitária e Ambiental da Universidade Federal do \\ PARÁ - UFPA, Campus de Tucuruí. \\ ${ }^{4}$ Prof. Mestre, Assistente II da Universidade Federal do PARÁ - UFPA, Campus de \\ Altamira.
}

Recebido em: 05/12/2014 - Aprovado em: 14/12/2014 - Publicado em: 15/12/2014

\section{RESUMO}

Este trabalho teve como objetivo, avaliar o teor de matéria orgânica e $\mathrm{pH}$ do solo em três sistemas agroflorestais (SAF's) com diferentes idades, o solo estudado é classificado como latossolo amarelo de textura argilosa para os três SAF's, a área experimental situa-se no município de Altamira na fazenda Guariba. Os períodos de coleta foram realizados no mês de abril de 2010 (período chuvoso) e setembro de 2010 (período seco). As coletas de amostra de solo foram efetuadas nas profundidades de 0-20 e 20-40 cm, sendo cada tratamento composto por três repetições. As amostras foram encaminhadas para os laboratórios de solo da Universidade Federal da Amazônia (UFRA) e laboratório de solo da EMBRAPA/CPATU - Belém-PA. Os resultados foram submetidos à análise estatística pelo programa SYSTAT 11. A pesquisa permitiu concluir que: $0 \mathrm{pH}$ do solo apresentou igualdade entre as profundidades, porém, foi diferente apenas para o período chuvoso e os tratamentos estudados; a matéria orgânica do solo é igual para os tratamentos e sazonalidade estudados, apenas foi diferente estatisticamente para as profundidades. Portanto, os sistemas agroflorestais estudados são ecossistemas que conservam de forma eficiente a matéria orgânica no solo, aumentando o reservatório de nutrientes neste recurso natural, sendo por isso, recomendado para a sustentabilidade de solos tropicais.

PALAVRAS-CHAVE: Matéria orgânica, Sistemas Agroflorestais; Solo.

\section{EVALUATION OF ORGANIC MATTER AND pH IN SOIL AGROFORESTRY LOCATED IN ALTAMIRA -PA}

\begin{abstract}
This study aimed to evaluate the organic matter content and soil $\mathrm{pH}$ in three agroforestry systems (SAF 's) of different ages, the studied soil is classified as yellow latosol clayey for the three SAF 's, the experimental area is located in the Altamira in Guariba farm. The collection periods were carried out in April 2010 (rainy season) and September 2010 (dry season). Soil sample collections were made the
\end{abstract}


depths of $0-20$ and $20-40 \mathrm{~cm}$, and each treatment consists of three replicates. The samples were sent to the soil laboratory of the Federal University of Amazonia (UFRA) and soil laboratory of EMBRAPA / CPATU - Belém- PA. The results were statistically analyzed by SYSTAT 11 . The research program shows that : the $\mathrm{pH}$ of the soil showed equality between depths, however, was different only for the rainy season and the treatments ; the soil organic matter is equal to the treatments studied and seasonality, was only statistically different for the depths. Therefore, the studied agroforestry systems are ecosystems that retain efficiently the organic matter in the soil, increasing the nutrient reservoir in this natural resource and is therefore recommended for the sustainability of tropical soils .

KEYWORDS: agroforestry systems; Solo and Organic Matter.

\section{INTRODUÇÃO}

A Região da Transamazônica, situada no sudoeste do estado do Pará, tem como característica inerente a agricultura familiar, que se caracteriza pela diversidade social, cultural e econômica dos agricultores, bem como dos seus sistemas de produção. O município de Altamira, atualmente conta com mais de 105 mil habitantes, possuindo em torno de $14,24 \%$ da população vivendo na zona rural (14.962 mil habitantes), desenvolvendo diferentes atividades: extrativismo, cultivos anuais e/ou perenes, criação de bovinos e/ou pequenos animais, o que vale ressaltar a importância da participação da agricultura familiar no sistema de produção rural do Estado do Pará (IBGE, 2010).

O manejo de derruba e queima em longo prazo promovem no solo alterações que os leva a degradação e, consequentemente, à impossibilidade de cultivos produtivos. Desta forma, o sistema agroflorestal pode ser usado permanentemente, minimizando a necessidade de derruba e queima de novas áreas e aumentando as chances de fixação do homem no campo, sendo uma alternativa para aproveitamento de áreas já alteradas ou degradadas (SILVA, 2011).

Os SAF's são considerados alternativas de uso sustentável do solo e do meio ambiente, capazes de alavancar os níveis de produtividade das lavouras de pequenos agricultores o qual propicia melhorias na qualidade do solo. A inclusão de componentes arbóreos pode manter ou aumentar a produtividade de determinada área, devido aos processos que aumentam ou reduzem perda no solo, como matéria orgânica, nutrientes e água, além de melhorar as propriedades do solo. E desta forma, os sistemas agroflorestais busca aumentar o teor de matéria orgânica e adicionando grande quantidade de biomassa e consequentemente protegendo 0 solo contra efeitos erosivos e elevação da temperatura (FERREIRA et. al, 2004; CARDOSO et. al, 2005)

Desta forma, torna-se necessário estudos relacionados à realidade enfrentada pelos agricultores, visando conhecer quais os principais entraves na adoção dos sistemas agroflorestais. Portanto, esta pesquisa teve como objetivo avaliar o teor da matéria orgânica (MO) e o pH do solo em diferentes Sistemas Agroflorestais, em função da sazonalidade e profundidade do solo, observando os SAF's como uma ferramenta fundamental para direcionar práticas que reduzam 0 seu depauperamento do solo. 


\section{MATERIAL E MÉTODOS}

O estudo foi realizado na fazenda Guariba, situada no município de Altamira, o qual pertence a messoregião Sudoeste Paraense e a microrregião Altamira. A sede

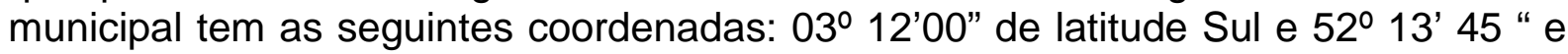
longitude a Oeste de Greenwich (IDESP, 2011).

O município possui área territorial de aproximadamente $159.695 .938 \mathrm{Km}^{2} \mathrm{e}$ tem a Rodovia Transamazônica (BR 230) como principal eixo integrador. A região de estudo possui o clima classificado como Af3, segundo o método de classificação de Köppen, sendo o solo estudado classificado como latossolo amarelo de textura argilosa para os três SAF's.

O delineamento experimental foi inteiramente casualizado (DIC), composto por três tratamentos, divididos em duas subparcelas (profundidades de 0-20 e 20-40 $\mathrm{cm}$ ), sendo que cada tratamento com três repetições. O SAF I foi designado para o sistema agroflorestal com aproximadamente 30 anos (consorciado por essências florestais, frutíferas e madeira de lei); o SAF II designado para o sistema agroflorestal tendo a origem da área com vegetação de floresta secundária, o qual foi enriquecido por diferentes espécies com quatro anos de idade (consórcio de essências florestais e frutíferas) e o SAF III designado para o sistema agroflorestal de aproximadamente um ano, a área antecede de vegetação de floresta secundária assim como o SAF II, neste o preparo da área foi mecanizada tendo como cultura principal o açaí consorciado com o mogno. Os SAF's I e II foram implementados com o cacau (cultura principal) e mantidas as espécies da área (florestais e frutíferas).

As amostras do solo foram coletadas e enviadas para as análises no Laboratório de Solos da Universidade Federal Rural da Amazônia, UFRA e da EMBRAPA Amazônia Oriental. As coletas foram realizadas em duas épocas distintas, sendo a $1^{\text {a }}$ coleta realizada em abril (período chuvoso) e a $2^{\text {a }}$ coleta no mês setembro (período seco) ambas em 2010. Em 2010 as áreas de estudos apresentavam 30, quatro e um ano, respectivamente nos SAF's I, II e III.

As variáveis analisadas como indicadores foram $\mathrm{pH}$ e Matéria Orgânica, os dados obtidos foram analisados pelo programa SYSTAT 11. Os dados foram submetidos a análises de ANOVA, sendo utilizados os teste T e F.

\section{RESULTADOS E DISCUSSÕES}

\section{- Profundidade}

A pesquisa mostrou que os valores de $\mathrm{pH}$ do solo, nas profundidades estudadas foram iguais para todos os tratamentos ( 0 a 20: Média $=5,7, \mathrm{DPM}= \pm$ 0,52; 20 a 40: Média= 5,6, DPM $= \pm 0,56$ ) conforme apresentado na Figura 1. SILVA (2011) estudando sistemas agroflorestais submetidos a queima e não queima, no município de Bragança-PA, corroboram com os resultados obtidos nesta pesquisa, onde relata-se que não houve diferença significativa para as profundidades de 5-10 e $10-20 \mathrm{~cm}$ variando o $\mathrm{pH}$ de 4,53 a 4,61. VASCONCELOS (2010) obteve resultados semelhantes estudando os SAFs no município de Igarapé-açu, com o pH variando de 4,8 a 5,28. Esses valores são classificados com acidez elevada, segundo OSAKI (1991) e RAIJ et al. (1996). Estudo realizado por BERTALOT (1997) com sistemas agroflorestais de dois anos de idade em Botucatu - SP, relata valores de $\mathrm{pH}$ variando de 4,9 a 5,5 na profundidade de $0-20 \mathrm{~cm}$. 


\section{$\mathrm{pH}$}

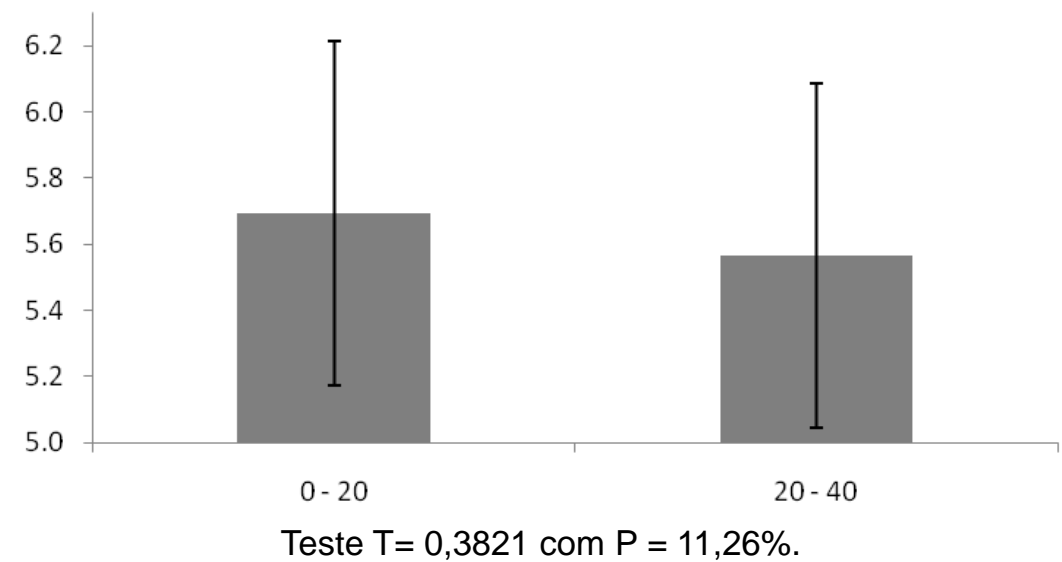

FIGURA 1: Valores de $\mathrm{pH}$ no período chuvoso, relativa à profundidade $0-20$ e $20-40 \mathrm{~cm}$ do solo.

Estudos realizados por REIS (2005) confirmam valores elevados de $\mathrm{pH}$, nas camadas superficiais em sistemas agroflorestais sob solo classificado como Latossolo Amarelo, no município de Marabá-Pa, ligados a presença de cátions básicos provenientes da decomposição da matéria-orgânica.

Analisando a matéria orgânica do solo observou-se que existe diferença significativa entre as profundidades em todos os tratamentos, ( 0 a 20: Média $=27,12$, $\mathrm{DPM}= \pm 6,40 ; 20$ a 40: Média= 18,33, $\mathrm{DPM}= \pm 7,19$ ) conforme a Figura 2. Resultados corroboram com os obtidos por SILVA (2011) que ratifica que independente do período de coleta, valores encontrados de matéria orgânica decresceram significativamente com a profundidade.

A MOS também tem grande participação na capacidade de tamponamento da solução no solo. Essa característica está relacionada à elevada CTC do húmus e à baixa dissolução de íons $\mathrm{H}+$ dos ácidos orgânicos. Assim sendo, o poder tampão é diretamente proporcional ao teor de MOS, ou seja, quanto maior o teor desta, maior será a resistência do solo à mudança de pH (SOUZA NETO, 2013).

\section{MO}

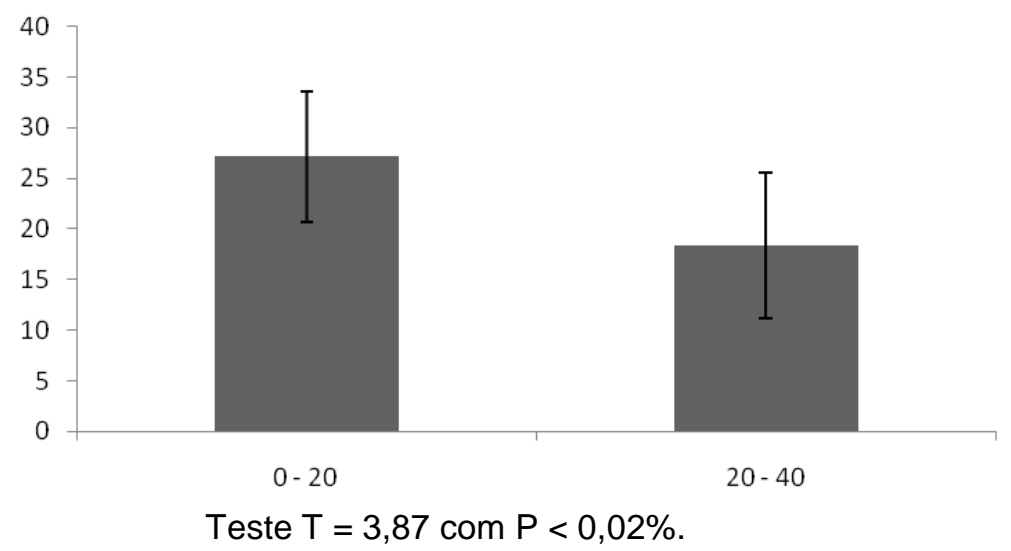

FIGURA 2: Teores de $M O$ no período chuvoso e seco, relativo as profundidades $0-20$ e $20-40 \mathrm{~cm}$. 
Pesquisa realizada por NEVES (2013) confirma que os maiores valores de matéria orgânica do solo foram encontrados nos horizontes superficiais do solo, decrescendo com o aumento da profundidade. A distribuição de matéria orgânica no perfil do solo depende, entre outros fatores, da forma de disposição desse material orgânico (RUIVO et al., 2005). A manutenção da matéria orgânica é essencial para sustentabilidade da agricultura, uma vez que os aumentos nos seus níveis conduzem, geralmente, a uma maior produtividade das culturas, e aumento na eficiência de utilização dos nutrientes (MALHI et al., 2011; PAUL et al., 2013).

- Sazonalidade

Houve diferença significativa entre os períodos chuvoso e seco respectivamente (Média $=5,81 \mathrm{com} D P M= \pm 0,65$; Média $=5,45 \mathrm{com} D P M= \pm 0,25$ ) para os valores de $\mathrm{pH}$ do solo em todos os tratamentos e profundidades, conforme a Figura 3.

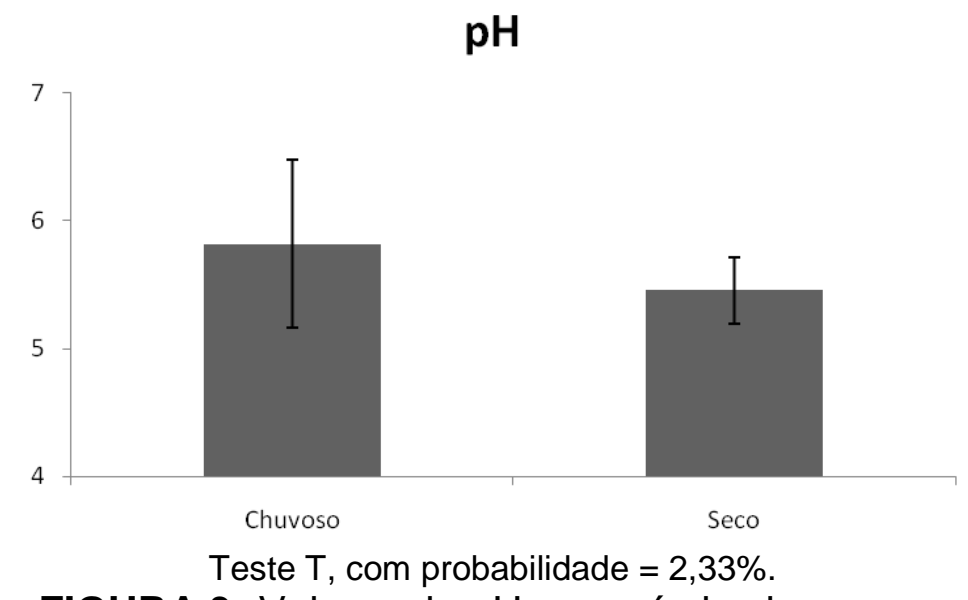

FIGURA 3: Valores de pH no período chuvoso e seco, relativa a sazonalidade

Observando a época de coleta do solo, nota-se que nesta pesquisa os maiores valores foram obtidos na época chuvosa, esses dados são semelhantes aos encontrados por SENA et al. (2006) em estudos com sistemas agroflorestais realizados em Marituba-PA, no entanto divergem dos obtidos por SILVA (2011) em estudos feitos em SAF's jovens em Bragança-PA, o qual obteve maiores valores de $\mathrm{pH}$ no período seco.

Para os teores de $\mathrm{MO}$, houve diferença significativa entre os períodos chuvoso e seco respectivamente (Média=27,09 com DPM= $\pm 6,52$; Média=18,36 com DPM= $\pm 6,75$ ) para todos os tratamentos e profundidades, conforme ilustra a Figura 4. 
MO

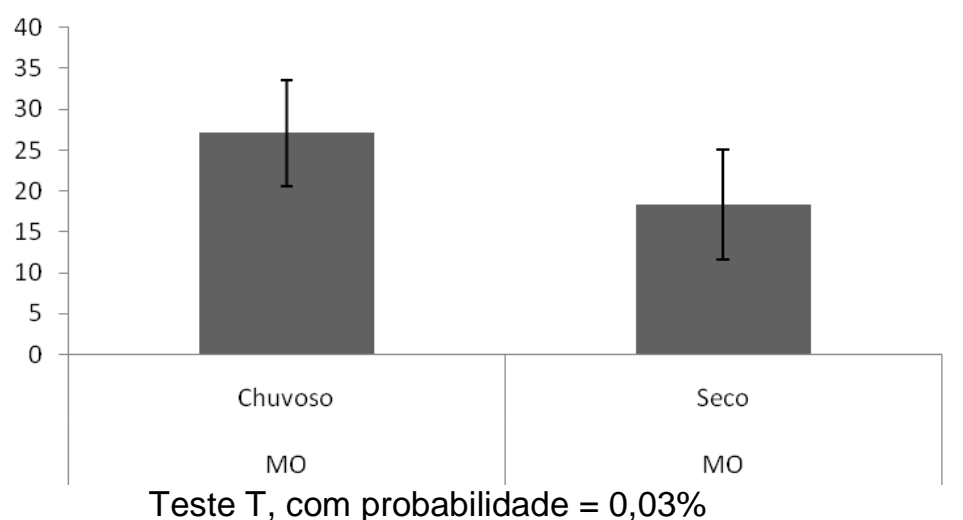

FIGURA 4: Teores de MO no período chuvoso e seco, relativa a sazonalidade.

A precipitação pluviométrica é um componente que controla, em escala regional, o processo de decomposição da matéria orgânica do solo (BERG, 2000). Para SILVEIRA et al., (2010) a adoção de sistemas conservacionistas, como o plantio direto e os sistemas agroflorestais, tem se apresentado como alternativa viável para assegurar a sustentabilidade do uso agrícola do solo, principalmente dos Latossolos, na região da Amazônia Brasileira.

- Sistemas Agroflorestais

Para os valores de pH, constatou-se que os SAF's I e III são iguais obtendo valores estatisticamente semelhantes (Média $=5,9 \mathrm{com} D P M= \pm 0,36$; Média $=6,4$ com $\mathrm{DPM}= \pm 0,46$ ) respectivamente, já o SAF's II obtém (Média $=5,08 \mathrm{com} \mathrm{DPM}= \pm$ $0,34)$ e é considerado diferente estatisticamente se comparado com o SAF's I e o III, (Figura 5) analisando o período chuvoso.

\section{pH (período chuvoso)}

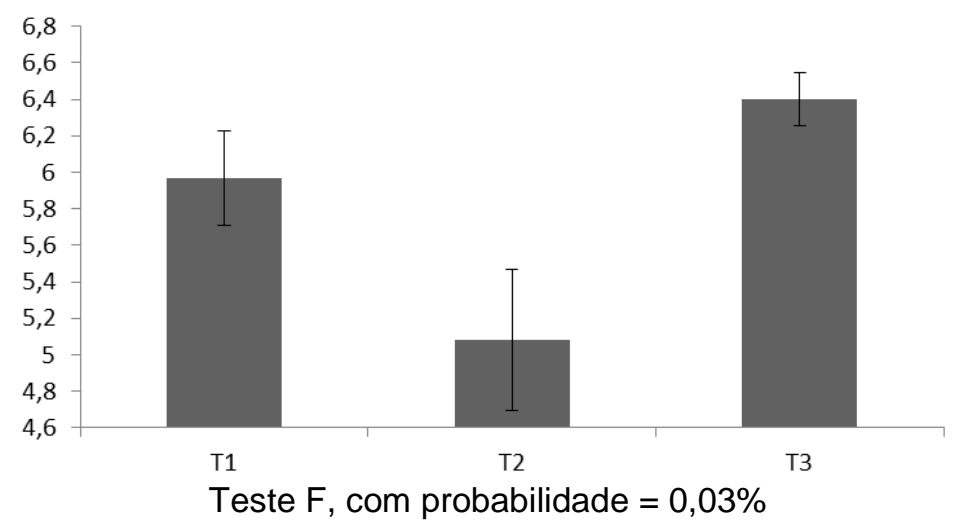

FIGURA 5: Valores de $\mathrm{pH}$ no período chuvoso, relativa ao tratamento.

Para melhor entendimento, pode-se afirmar que o SAF'l obteve esse valor de $\mathrm{pH}$ próximo de 6 por decorrência de ser um sistema agroflorestal mais antigo com idade aproximada de 30 anos, e para o SAF's III entende-se que a área por ter sido mecanizada, e a matéria orgânica ter sido incorporada ao solo, esse entrou em 
processo de decomposição fornecendo mais nutriente ao solo e consequentemente neutralizando os cátions ativos do mesmo.

Para o SAF's II por ter sido um sistema de enriquecimento de capoeira e pela idade de quatro anos aproximadamente, pode-se considerar que a idade pode ser um fator preponderante que vem influenciar nos resultados obtidos. Analisando o período seco Pode-se afirmar de os SAF's I, II e III são iguais estatisticamente para os valores de $\mathrm{pH}$, entre os diferentes tratamentos e profundidades estudados (Figura $6)$.

\section{pH (período seco)}

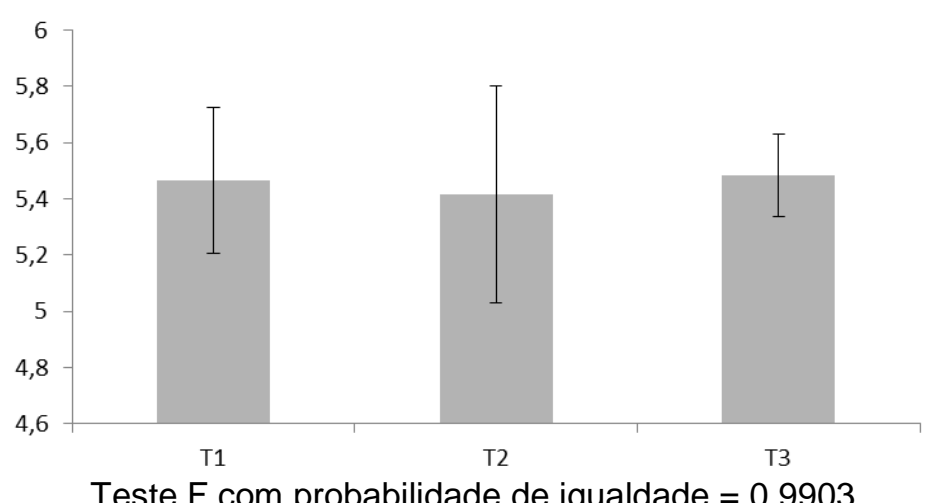

FIGURA 6: Valores de $\mathrm{pH}$ no período seco, relativa ao tratamento.

Para o MOS, pode-se afirma que os SAF's I, II e III, são iguais estatisticamente quando observados no período chuvoso (Figura 7A) e no período seco (Figura 7B). Pesquisa realizada por SILVA (2011) mostrou que os valores obtidos de $\mathrm{MO}$ em função dos diferentes sistemas agroflorestais submetidos a queima e sem queima, apresentaram teores estatisticamente semelhantes aos encontrados na Floresta Secundária. Mesmo em solo de baixa fertilidade, florestas exuberantes não apresentam sintomas de deficiência nutricional, uma vez que o ciclo de nutrientes é praticamente fechado, verificando-se, ao longo do ano, contínua a decomposição do material orgânico, associada a pequena perda por lixiviação e absorção de elementos.

Nos sistemas agrícolas, o manejo adotado tem grande influência nos estoques de MOS, podendo diminuir, manter ou aumentar esses estoques em relação à vegetação nativa (KHORRAMDEL et al., 2013). 

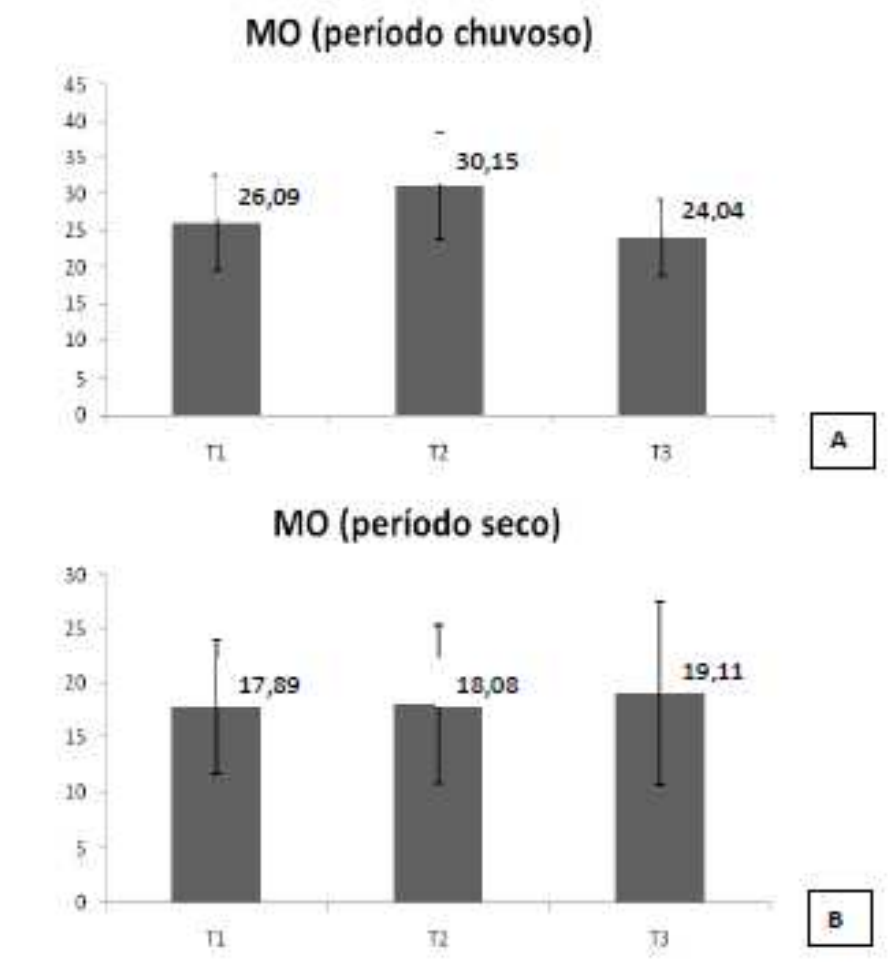

Teste $\mathrm{F}$ com probabilidade $=0,9543(\mathrm{~A})$ e de $=0,1702(\mathrm{~B})$

FIGURA 7: Análise do $\mathrm{MO}$ no período chuvoso (A) e no período seco (B), em diferentes SAF's.

Estudos realizados por CALVI (2009) confirmaram que, existem várias experiências de SAF's desenvolvidas por produtores familiares na Transamazônica, além de projetos e programas executados por diferentes organizações e instituições que buscam sensibilizar os agricultores a implantarem os sistemas agroflorestais. $O$ autor relata que essas ações ainda não atingiram um público expressivo, ressaltando que os SAF's mesmo apresentando características comprovadamente vantajosas, percebe-se índice de adoção bastante reduzido se comparado com o universo da agricultura familiar deste território.

Portanto, vale ressaltar que a utilização de SAFs também é considerada como benefício na recuperação de áreas degradadas, apresentando resultados positivos para a melhoria das propriedades físicas e químicas dos solos, assim como propiciam condições mais favoráveis para o estímulo da atividade dos microrganismos; esta é resultante, principalmente, do grande aporte de matéria orgânica ao solo (BRANDÃO, 2013).

\section{CONCLUSÃO}

Os pHs do solo foram iguais entre as profundidades, apenas foi diferente estatisticamente entre os períodos chuvoso e entre os tratamentos estudados, sendo que apresenta valores mais elevados. A matéria Orgânica do solo não apresentou diferença entre os tratamentos estudados e sazonalidade, altera somente para a profundidade.

Os sistemas agroflorestais estudados permitem indicar que este modelo é um ecossistema que conserva de forma eficientemente a matéria orgânica no solo, 
aumentando o reservatório de nutrientes neste recurso natural, sendo por isso, recomendado para a sustentabilidade de solos tropicais..

\section{REFERÊNCIAS}

BERG, B. Litter decomposition and an organic matter turnover in northern forest soils. For. Ecol. Manag., v. 133, p. 13-22, 2000.

BERTALOT, M. J. A. Crescimento e avaliação nutricional de leguminosas arbóreas potenciais para ecossistemas Agroflorestais num solo de Cerrado. Dissertação (Mestrado). Universidade Estadual Paulista, Botucatu-SP, 1997. 63p.

BRANDÃO, F. J. C. Caracterização de um Latossolo em sistemas agroflorestais e mata com o uso de análise estatística multivariada. Tese (doutorado) Universidade Estadual Paulista, Faculdade de Ciências Agrárias e Veterinárias. Jaboticabal, 2013.

CALVI, M. F. Fatores de adoção de sistemas agroflorestais por agricultores familiares do Município de Medicilândia, Pará. 2009. 122 f. Dissertação (Mestrado em Agriculturas Familiares e Desenvolvimento Sustentável) Universidade Federal do Pará, Núcleo de Ciências Agrárias e Desenvolvimento Rural, Programa de PósGraduação em Agriculturas Amazônicas, Belém-Pará. 2009. p15.

CARDOSO, I. M.; SOUZA, H. N.; MENDONÇA, E. S. Biodiversidade, recursos genéticos e cuidados fitossanitários. R. Ação Ambiental, V. 31, p. 18-20, 2005.

FERREIRA, C. P.; KATO, O. R; COSTA, C. A. C. carbono orgânico, nitrogênio e a razão $\mathrm{C} / \mathrm{N}$ em solo sob sistemas agroflorestais no nordeste paraense, In: CONGRESSO BRASILEIRO DE SISTEMAS AGROFLORESTAIS, 5, 2004, Curitiba, CD-Rom, Anais... EMBRAPA. 2004.

IBGE - INSTITUTO BRASILEIRO DE GEOGRAFIA E ESTATÍSTICA. Disponível em: $<$ http://www.ibge.gov.br/home/estatistica/indicadores/agropecuaria/lspa/>. Acessado em 28 de dezembro de 2010.

IDESP - INSTITUTO DE DESENVOLVIMENTO ECONOMICO, SOCIAL E AMBIENTAL DO PARÁ. Estatística Municipal: Altamira, 2011. Disponível em: http://iah.iec.pa.gov.br/iah/fulltext/georeferenciamento/altamira.pdf. Acessado em: em 28 de dezembro de 2010

KHORRAMDEL, S.; KOOCHEKI, A.; MAHALLATI, M.N.; KHORASANI, R.; GHORBANI, R. Evaluation of carbon sequestration potential in corn fields with different management systems. Soil \& Tillage Research, v.133, p.25-31, 2013.

MALHIA, S.S.; NYBORG. M.; SOLBERG, E.D.; DYCK, M.F.; PUURVEEN, D. Improving crop yield and $\mathrm{N}$ uptake with long-term straw retention in two contrasting soil types. Field Crops Research, v.124, p.378-391, 2011. 
NEVES, Y. Y. B. Características de diferentes sistemas de uso do solo em Cruzeiro do Sul, Acre. Dissertação (mestrado) - Universidade Federal de Lavras, Lavras: UFLA, 2013. 103p.

PAUL, B.K.; VANLAUWE, B.; AYUKE, F.; GASSNER, A.; HOOGMOED, M.; HURISSO, T.T.; KOALA, S.; LELEI, D.; NDABAMENYE, T.; SIX, J.; PULLEMAN, M. $M$. Medium-term impact of tillage and residue management on soil aggregate stability, soil carbon and crop productivity. Agriculture, Ecosystems and Environment, v.164, p.14-22, 2013.

OSAKI, F. Calagem e adubação. Campinas. Instituto Brasileiro de Ensino Agrícola, 1991. $503 \mathrm{p}$.

RAIJ, B. V.; CANTARELA, H.; QUAGGIO, J. A.; FURLANI, A. M. C. Recomendações de adubação e calagem para o Estado de São Paulo. Campinas. Instituto Agronômico \& Fundação IAC, 1996. 285 p. (Boletim Técnico, 100).

REIS, M. da S. Composição granulométrica e características químicas do solo de uma toposseqüência sob pastagem, numa frente pioneira da microrregião de Marabá-PA. 2005. p. 71. Dissertação (Mestrado). Universidade Federal Rural da Amazônia - UFRA, Belém-PA. 2005.

RUIVO, M. L. P.; AMARAL, I. G.; FARO, M. P. da S.; RIBEIRO, E. L. C. GUEDES, A. L. S.; SANTOS, M. M. de L. S. Caracterização química da manta orgânica leve em diferentes tipos de solo em uma toposseqüência na ilha de Algodoal/Maiandeua, PA. Bol. Mus. Para. Emílio Goeldi, Série. Ciências Naturais, v. 1, n. 1, p. 227-234, abr. 2005.

SENA, W. de L. Avaliação dos atributos químicos e carbono microbiano de um Latossolo Amarelo sob sistemas agroflorestais em comparação coma floresta secundária, Marituba, Pará. 2006. 103 p., Tese (Doutorado), Universidade Federal Rural da Amazônia e Embrapa- Amazônia Oriental, Belém-PA, 2006.

SOUSA NETO, O. N. de. Análise multivariada dos atributos físicos e químicos de um Cambissolo cultivado sob práticas de manejo sustentável da caatinga. Dissertação (Mestrado em Ciência do solo) Área de concentração em Manejo e conservação do solo - Universidade Federal Rural do Semi-Árido. Mossoró, 2013.

SILVA, S. A. S da. Avaliação dos atributos químicos e microbianos em latossolo amarelo sob sistema agroflorestal e floresta secundária em Bragança, Pará. Tese (Doutorados). 2011. Universidade federal Rural da Amazônia - UFRA, Belém - PA, 2011. 97 p.

SILVEIRA, P. M. da; CUNHA, P. C. R. da; STONE, L. F.; SANTOS, G. G. dos. Atributos químicos de solo cultivado com diferentes culturas de cobertura. Pesq. Agropec. Trop., Goiânia, v. 40, n. 3, p. 283-290, jul./set. 2010.

VASCONCELOS, J. M. Indicadores químicos e biológicos de latossolo amarelo submetido ao sistema de preparo de área usando a queima e trituração da AGRARIAN ACADEMY, Centro Científico Conhecer - Goiânia, v.1, n.02; p. 24 2014 
vegetação no Nordeste Paraense. 2010. p.105. Dissertação (Mestrado), Universidade Federal Rural da Amazônia, Belém-PA, 2010. 